\title{
PRONTUÁRIOS, PARA QUE SERVEM? REPRESENTAÇÃO DOS COORDENADORES DE EQUIPE DOS CAPSi A RESPEITO DO VALOR E DA UTILIDADE DOS PRONTUÁRIOS
}

\author{
PATIENTS RECORDS, WHAT ARE THEY USED FOR? \\ REPRESENTATION OF CAPSIS' TEAM COORDINATORS ABOUT \\ THE VALUE AND USE OF PATIENTS RECORDS
}

\author{
Alberto Olavo Advincula Reis ${ }^{1}$ \\ Caroline Dombi-Barbosa ${ }^{2}$ \\ Moacyr Miniussi Bertolino Neto ${ }^{3}$ \\ Maria Margarida Licursi Prates ${ }^{4}$ \\ Patrícia Santos de Souza Delfini ${ }^{5}$ \\ Felipe Lessa Fonseca ${ }^{6}$ \\ Ariana Queiroz de Oliveira ${ }^{7}$
}

Reis AOA et al. Prontuários, para que servem? Representação dos coordenadores de equipe dos CAPSI a respeito do valor e da utilidade dos prontuários. Rev Bras Crescimento Desenvolv Hum. 2009; 19(3): 383-392.

\section{Resumo:}

Introdução: o artigo procura compreender como os Coordenadores dos Centros de Atenção Psicossocial Infantojuvenil (CAPSi) do Estado de São Paulo percebem o valor e a utilidade dos prontuários do usuários do serviço. Atualmente, os prontuários obedecem a claros princípios éticos. São muitas as pesquisas que se utilizam de prontuários e vários os estudos que se propõem a aperfeiçoá-los uma vez que são considerados ferramenta de primeira importância no âmbito da saúde. Cabe, entretanto, indagar se esses instrumentos são valorizados e se suas utilidades percebidas pelas equipes de saúde mental no exercício real de suas práticas. O trabalho descreve e analisa as percepções dos Coordenadores dos CAPSi-SP a respeito da utilidade dos prontuários para a equipe de saúde e para os usuários. Método: foi entrevistado um Coordenador de cada um dos 19 dos CAPSi do Estado de São Paulo. As respostas foram analisadas de acordo com os procedimentos clássicos da Análise de Conteúdo. Resultados: os prontuários são percebidos como valiosos instrumentos de trabalho e sua importância destacada como instrumento de intervenção e de acompanhamento clínicos. Sua relevância é assinalada como dispositivo que possibilita a articulação e a comunicação dos membros das equipes técnicas dos CAPSi. Por outro lado, não é percebida qual a utilidade que eles teriam para o usuário. A contradição nos níveis de importância alocada à utilidade dos prontuários quando se trata da equipe técnica ou dos usuários enseja uma discussão aprofundada sobre a natureza da clínica em saúde mental praticada nos CAPSi.

Palavras-chave: CAPSi; prontuários; criança/adolescente; saúde mental.

Trabalho resultante de processo FAPESP nº 2006/06902-2, realizado pelo Laboratório de Saúde Mental Coletiva (LASAMEC), da Faculdade de Saúde Pública da USP.

1 Professor Doutor do Dept. de saúde materno-infantil da Faculdade de Saúde Pública da USP, orientador de Pós-graduação do Programa de Saúde Pública da Faculdade de Saúde Pública da USP. Coordenador do grupo de pesquisa CNPq do Laboratório de Saúde Mental Coletiva - LASAMEC. E-mail: albereis@usp.br

2 Psicóloga. Mestre em Saúde Pública. do depto. de Saúde Materno-Infantil da Faculdade de Saúde Pública da USP. Pesquisadora do LASAMEC. E-mail: caroldombi@usp.br

3 Psicólogo, pesquisador, membro do LASAMEC. E-mail:

4 Terapeuta Ocupacional, pesquisadora, membro do LASAMEC.

5 Psicóloga. Mestranda do Depto. de Saúde Materno-Infantil da Faculdade de Saúde Pública da USP. Pesquisadora do LASAMEC. Bolsista CNPq - Brasil. E-mail: patriciadelfini@usp.br

6 Psicólogo. Doutor pela Pontifícia Universidade Católica/SP. Pesquisador do LASAMEC. E-mail: flessaf@uol.com.br

7 Psicóloga, Mestranda da UNIFESP, membro do LASAMEC. E-mail: arianapsi@uol.com.br 


\begin{abstract}
:
Introduction: the article aims to understand how the coordinators of Psychosocial Care Centres for Children and Adolescents (CAPSi) from the state of São Paulo perceive the value and use of patients records. Nowadays, patients records obey clear ethical principles. There are several studies that collect data from patients records and others proposed to provide their improvement, because they are considered important tools in health system. However, it is important to ask if these tools are valuable and if their use is perceived by mental health teams in their everyday practices. The article describes and analyses the perception of CAPSi's coordinators about the use of records for teams and patients. Method: One coordinator from each of the 19 CAPSi from the state of São Paulo were interviewed. The answers were analysed according to the classic procedures of content analysis. Results: the records are perceived as valuable work tools and they are considered important as intervention and clinical follow-up tools. Their relevance is also related as a tool that makes the articulation and communication among workers from CAPSi possible. On the other hand, the use they should have for patients is not perceived. The contradiction between levels of importance of the tool for workers and patients may point to the importance of a discussion about the mental health clinic practiced in CAPSi.
\end{abstract}

Key words: CAPSi; patients records; children/adolescent; mental health.

\section{INTRODUÇÃO}

Nem sempre a existência de prontuários, que acompanham a trajetória dos usuários dos equipamentos de saúde mental e nos quais se inscrevem os diversos dados, ações, cuidados e medidas atinentes a esses sujeitos, foi reconhecida como sendo de real utilidade para eles, nem sua existência representou um valor positivo por parte de setores de trabalhadores da saúde mental. Já se argumentou criticamente, num momento histórico em que as instituições de saúde mental respondiam maciçamente ao modelo médico-hospitalar, que prontuários cristalizavam a vida e enrijeciam a dinâmica da situação dos sujeitos que neles tinham suas vicissitudes inscritas e, assim, contribuíam para a reificação e estigmatização dos processos que os afetavam. A existência dessa modalidade de registro prender-se-ia tão exclusivamente a medidas burocrático-administrativas, não raro ideológicas e derivadas mimeticamente de procedimentos próprios da área que se ocupa das doenças físicas. Parte dessas críticas foi derivada dos estudos interacionista-simbólicos de
Goffman $^{1-3}$, das asserções antipsiquiátricas oriundas diretamente das obras de Laing ${ }^{4}$ e Cooper ${ }^{5}$ ou de sua recuperação pela psicanálise lacaniana na versão de Maud Mannoni6 ${ }^{6}$. De acordo com essa perspectiva, a dinâmica da saúde mental, em virtude da natureza específica do transtorno mental, prescindiria do recurso de prontuários.

Atualmente, com a saúde mental fundada em outras bases e que em parte se inspiraram dessas críticas, os prontuários e seus usos obedecem a claros princípios que fazem do usuário seu proprietário e das instituições seu guardião, consoante a princípios éticos bem delineados. Exemplo disso é o que reza o artigo 70 do Código de Ética Médico ${ }^{7}$, e de acordo com o qual se torna vedado ao médico " $\mathrm{Ne}$ gar ao paciente acesso a seu prontuário médico, ficha clínica ou similar, bem como deixar de dar explicações necessárias à sua compreensão, salvo quando ocasionar riscos para o paciente ou para terceiros." Este direito é geralmente denominado de "habeas data". Dessa forma, mesmo que ainda não totalmente compreendido por alguns profissionais, o pron- 
tuário é de total propriedade do paciente, tendo este o direito de acessá-lo, a qualquer momento, bem como o paciente tem o direito de negar o acesso ao seu prontuário por qualquer profissional desde que assim o deseje"8 (Costa, 2001:55).

Dentre os elementos que participaram dessa inflexão destacam-se a transformação da natureza do sistema de saúde mental, a emergência de novos equipamentos sociais de atenção tais como os Centros de Atenção Psicossocial e as Residências Terapêuticas, aliados a uma compreensão baseada em princípios éticos de natureza democrático-cidadã dos prontuários.

Nesses termos, parece existir consenso sobre a importância dos prontuários seja para seguimento, segurança e respaldo do usuário e suas utilizações são diversas. Assim, são muitas as pesquisas que se utilizam de prontuários e são vários os estudos e ensaios que se propõem a aperfeiçoar e modernizar os prontuários considerados então como uma ferramenta de primeira importância no âmbito da saúde mental pública ${ }^{9,10}$. Nas resoluções do Conselho Federal de Medicina referente ao prontuário, definido como "conjunto de documentos padronizados e ordenados, destinados ao registro dos cuidados profissionais ao paciente pelos serviços de saúde públicos ou privados"11 existe o reconhecimento do valor do prontuário para o paciente, a instituição que o atende, bem como para o médico, o ensino, a pesquisa e os serviços de saúde pública.

À luz dessas considerações, poder-se-ia perguntar se os princípios éticos que regem os prontuários não se constituiriam apenas como superestruturas desconectadas do saber comum que organiza, de fato, a realidade das práticas dos serviços de saúde mental. Se assim for, qual seria então o valor que as equipes de saúde mental alocam aos prontuários no exercício real de suas práticas cotidianas? Quais são as utilidades percebidas por essas equipes no tangente aos prontuários?
O presente trabalho, em face de tais interrogações, descreve e analisa as percepções que os Coordenadores dos CAPSi do Estado de São Paulo têm a respeito da utilidade dos prontuários. A pergunta indutora e as respostas que dela decorreram permitiram que se explorassem as percepções a respeito das diversas modalidades de utilidades que os prontuários encerram. Essas percepções foram estudadas a partir de duas perspectivas: a utilidade para os usuários e a utilidade para a equipe técnica.

\section{MÉTODO}

Os resultados apresentados nesse artigo remetem-se à análise das respostas a uma questão (subdividida em duas perguntas) de um total de sete questões abertas que compuseram uma entrevista estruturada aplicada aos Coordenadores dos 19 CAPSi do Estado de São Paulo, cadastrados há pelo menos um ano até 2006. A entrevista constitui parte da pesquisa “Caracterização Epidemiológica e Sóciodemográfica dos Centros de Atenção Psicossocial Infanto-juvenis (CAPSi) no Estado de São Paulo”, processo Fapesp n 2006/ 06902-2, aprovada pelo Comitê de Ética da Faculdade de Saúde Pública da USP (protocolo $n^{\circ} 1616$ de 20 de abril de 2007).

Escolheu-se os Coordenadores de Equipe como sujeitos da investigação pelo fato deles assumirem função de liderança tanto nas atividades atinentes aos projetos terapêuticos como de responsabilidade na manutenção de registro de produtividade e de interlocução com os diferentes atores que atuam junto aos CAPSi. As respostas foram gravadas e transcritas, sendo, então, objeto de análise e categorização de acordo com os procedimentos clássicos da análise de conteúdo ${ }^{12-14}$. Um treinamento cuidadoso da equipe de entrevistadores, formada por mestrandos, doutorandos, profissionais da área da psicologia, terapia ocupacional, educação e 
enfermagem, aliado a um número conciso de perguntas permitiu que se reduzisse ao mínimo o viés de entrevistador, eventualmente capaz de afetar a qualidade das respostas.

A estrutura do instrumento voltado à coleta de informações comportou três temas,

1. Inserção dos usuários no CAPSI [ $1^{\text {a }}$ questão]

2. Processo de estabelecimento de diagnóstico [ $2^{\mathrm{a}}$ questão e subquestões]

3. Processo de registro e utilização dos prontuários [da $3^{\mathrm{a}}$ a $7^{\mathrm{a}}$ questão e subquestões].

As entrevistas foram agendadas e realizadas nos próprios CAPSi consoante à disponibilidade dos sujeitos. A questão ${ }^{\circ} 4$ foi retida para o presente trabalho e consistiu na seguinte formulação:

Questão 4 - Qual a utilidade dos prontuários para:

a. Equipe Técnica

b. Usuário

Para a análise das respostas foi realizada uma leitura geral (envolvendo as duas perguntas) tendo por crivo o valor do prontuário, uma vez que se observou que todas as respostas sobre a utilidade dos prontuários traziam consigo referências ao valor desses instrumentos. Assim, decidiu-se por reter todas as unidades de significações que, na percepção dos Coordenadores, se remetiam ao valor representado pelos prontuários para os CAPSi. Por último, foram sorteados aleatoriamente dois CAPSi, um do Interior e outro da Capital do Estado de São Paulo para que se extraíssem as expressões mais ilustrativas a respeito do valor dos prontuários emitidas por seus Coordenadores. Para o tratamento específico das respostas das duas perguntas procedeu-se a uma análise temática categorial. Para tanto, a totalidade das respostas foi lida, efetuando-se nesse processo ao recorte e estabelecimento de unidades de significação que se remetiam a duas categorias maiores definidas de antemão: a) utilidade para a equipe $\mathrm{e}$

b) utilidade para os usuários.

As unidades de significação, uma vez associadas por nexos lógicos de similaridade semântica, deram origem a diversas categorias. O procedimento que, em seguida, permitiu a construção e a hierarquização das categorias foi pautado por dois critérios:

1. aproximação das subcategorias que encerravam maior ou menor freqüência de unidades de significação de respostas (critério quantitativo);

2. relação de significação que cada uma da subcategoria mantém com as outras (critério qualitativo).

\section{RESULTADOS E DISCUSSÃO}

\section{Valor dos prontuários}

O valor dos prontuários foi considerado positivo para os Coordenadores, não havendo sido registrada nenhuma expressão, idéia ou menção a respeito de qualquer qualidade negativa a eles alocada. $\mathrm{O}$ conjunto das respostas indica a enorme importância que o prontuário encerra para as Equipes dos CAPSi. Frases e expressões, produzidas por representante de um CAPSi do Interior e outro da Grande São Paulo, como as abaixo transcritas retratam o conjunto das falas dos entrevistados:

"Ah! Eu acho que é de uma riqueza ímpar né? Porque cada um tem uma ação, então é só quem for atender aquele caso, seja a família, seja a escola; então é subsídio assim, importantíssimo, né? Você ter a fotografia daquele paciente, a dinâmica da família, todo dia dele, né? Muito importante." (CAPSi 12 Grande São Paulo)

"Para a Equipe é fundamental: é o instrumento de trabalho. Aqui a gente zela por um bom registro. Imagina só, esses dias veio uma mãe aqui, que a filha fez atendimento em 2004, e veja bem, se eu não tive um bom registro como é que eu vou poder fazer um 
bom relatório? A gente faz o registro de tudo, tudo mesmo, é bem elaborado e sobre todas as consultas, cada atividade. É o meio de comunicação da equipe, se a referência não tá aqui e alguma pessoa precisa de alguma informação vai recorrer ao prontuário." (CAPSi 9 - Interior)

A coincidência de percepção acerca do valor dos prontuários presente nas falas dos Coordenadores oriundos de duas realidades geodemográficas distintas indica que os prontuários são considerados como instrumento de trabalho e de cidadania importante para os CAPSi.

\section{A. Utilidade dos prontuários para as Equipes}

As primeiras Categorias obtidas a partir das Unidades de Significação acerca da percepção da utilidade dos prontuários para a EQUIPE foram:

- Instrumento de orientação de conduta;

- Base de atendimento;

- Canal de troca de informação entre os membros da equipe técnica;

- Lugar de aglutinação e ampliação da visão da Equipe;

- Inscrição da história da criança ou do adolescente nos CAPSi;

- Lugar de apropriação da história do sujeito;

- Instrumento com o qual a equipe pode contar para responder a alguma demanda ou questionamento vindo de fora do CAPSi;

- Referência para a equipe;

- Recurso de memória.

As Unidades de Significação que fizeram menção ao fato dos prontuários serem percebidos como "instrumento de avaliação do estado da criança ou do adolescente”, como "instrumento de orientação de conduta" e "base de atendimento" definiram a subcategoria:

\section{INSTRUMENTO DE INTERVENÇÃO} E DIAGNÓSTICO.

As referentes ao "canal de troca de informação entre os membros da equipe técnica”, "lugar de aglutinação e ampliação da visão da Equipe” deram lugar à categoria: BASE DE TROCAS DE INFORMAÇÃO.

Quando se tratava de falas que apontavam para o fato do prontuário "inscrever a história da criança ou do adolescente nos CAPSi" ou de ser "o lugar de apropriação da história do sujeito", elas foram organizadas sob a subcategoria SEDIMENTAÇÃO DA HISTÓRIA DO SUJEITO” e, finalmente, quando fizeram menção de que o prontuário é um instrumento com o qual a equipe pode contar para responder a alguma demanda ou questionamento vindo de fora do CAPSi ou quando é percebido como "referência para a equipe", "recurso de memória”, elas foram colocadas sob a subcategoria SEGURANÇA PARA EQUIPE.

Deste modo, pôde-se construir 4 subcategorias maiores:

1. Base de troca de informação entre os membros da equipe técnica;

2. Instrumento de intervenção e diagnóstico junto à criança/adolescente;

3. Sedimentação da história do sujeito;

4. Fator de segurança para a equipe.

Essas quatro subcategorias foram por sua vez aglutinadas em duas categorias mais gerais.

1. INTERVENÇÃO INTEGRAL englobou as subcategorias:

a. Base de troca de informação;

b. Instrumento de intervenção e diagnóstico.

2. DOCUMENTO IDENTITÁRIO que associou as subcategorias:

a. Sedimentação da História do Sujeito;

b. Segurança para a Equipe e o Paciente. 
Figura 1: Percepção da utilidade dos prontuários para a equipe segundo os Coordenadores dos CAPSi, Estado de São Paulo, 2009.

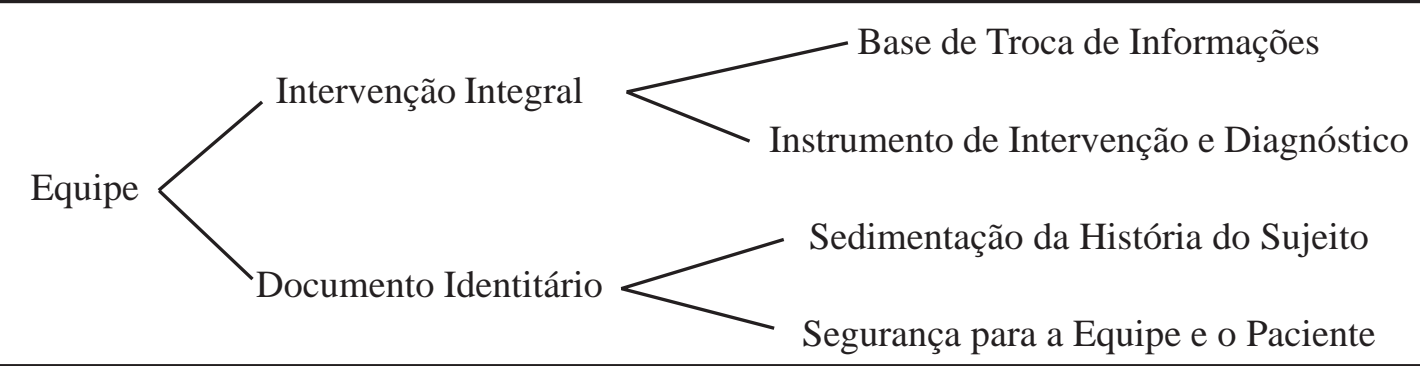

Fonte: REIS AOA. Pesquisa Caracterização Epidemiológica e Sócio-demográfica dos Centros de Atenção Psicossociais Infantojuvenis do Estado de São Paulo, processo FAPESP 06/06902-2.

A principal utilidade do prontuário é a de ser um Instrumento de Intervenção e Diagnóstico, representando $41,9 \%$ das respostas. Em seguida, situam-se as respostas que indicam ser os prontuários um Lugar de Troca de Informação entre os membros da Equipes (22,6,0\%) Sedimentação da História do Sujeito $(25,8 \%)$ e, por último, Lugar de Segurança $(9,7 \%)$. A freqüência acumulada dos dois tipos de respostas, "Instrumento de Intervenção e Diagnóstico" e "Lugar de Troca de Informação entre os membros da Equipe" evidencia a importância desse conjunto (64,5\%). Além, disso essas duas subcategorias entretêm entre si um nexo lógico que possibilita que formem juntas uma categoria maior.

As duas subcategorias articuladas entre si, sob a denominação Intervenção Integral, definem o núcleo central da significação relativa à utilidade do questionário para a equipe técnica. Considerando que a orientação e a intervenção diagnóstica devam ser, de acordo com os princípios dos CAPS e do SUS, conduzidas sob a lógica da integralidade e, consequentemente, da multidisciplinaridade que caracteriza o trabalho de uma equipe de saúde mental da rede pública, o fato do prontuário ter uma função integradora no âmbito da comunicação e da troca de informação entre os membros da equipe faz com que a articulação dessas duas subcategorias mostre, nesses termos, seu nexo lógico.
Resulta então poder afirmar que o prontuário tem sua maior importância como função operatória clínica. Outra utilidade percebida é a de assumir uma função psicossocial ao possibilitar encontro, interação, comunicação da equipe cujos membros pouco discutem ou nem sempre se encontram entre si.

A segunda categoria denominada DOCUMENTO IDENTITÁRIO foi formada a partir da associação das duas últimas subcategorias:

1. Lugar de sedimentação da história

2. Lugar de segurança

A associação entre as subcategorias Lugar de Sedimentação da história do sujeito e Lugar de Segurança para a equipe e o paciente permitiu a formação da categoria Documento Identitário. A freqüência das unidades de significação que deram origem à primeira representa $25,9 \%$ enquanto as que formaram a segunda. Lugar de Segurança representaram 9,7\% das respostas. O conjunto das duas subcategorias responde por $35,5 \%$ do total de todas as respostas. A significação comum entre elas se mostra no fato de que é pela apropriação e sedimentação da história do sujeito é que se pode responder a qualquer demanda ou questionamento oriundos da família, da justiça ou de diversas outras instituições ou grupos. 
Tabela 1: Distribuição do número e proporção das Unidades de Significação para as Categorias referentes à Utilidade dos Prontuários para a Equipe na percepção dos Coordenadores dos CAPSi. São Paulo, 2009.

\begin{tabular}{|c|c|c|c|c|c|}
\hline CATEGORIAS & SUBCATEGORIAS & frequência & $\begin{array}{l}\text { frequência } \\
\text { relativa }\end{array}$ & $\begin{array}{l}\text { frequência } \\
\text { acumulada }\end{array}$ & $\begin{array}{l}\text { frequência } \\
\text { relativa } \\
\text { acu]mulada }\end{array}$ \\
\hline \multirow{2}{*}{$\begin{array}{l}\text { INTERVENÇÃO } \\
\text { INTEGRAL }\end{array}$} & $\begin{array}{l}\text { Base de Troca de } \\
\text { Informações }\end{array}$ & 7 & 22,6 & 7 & 22,6 \\
\hline & $\begin{array}{l}\text { Instrumento de } \\
\text { Intervenção e } \\
\text { Diagnóstico }\end{array}$ & 13 & 41,9 & 20 & 64,5 \\
\hline \multirow{3}{*}{$\begin{array}{l}\text { DOCUMENTO } \\
\text { IDENTITÁRIO }\end{array}$} & $\begin{array}{l}\text { Sedimentação da } \\
\text { História do Sujeito }\end{array}$ & 8 & 25,8 & 28 & 90,3 \\
\hline & $\begin{array}{l}\text { Segurança para a } \\
\text { Equipe e o Paciente }\end{array}$ & 3 & 9,7 & 31 & 100,0 \\
\hline & TOTAL & $31^{*}$ & 100,0 & 31 & 100,0 \\
\hline
\end{tabular}

Fonte: REIS AOA. Pesquisa Caracterização Epidemiológica e Sócio-demográfica dos Centros de Atenção Psicossociais Infantojuvenis do Estado de São Paulo, processo FAPESP 06/06902-2. *O número de unidades de significação supera o número de entrevistados posto que um sujeito pode produzir mais de uma unidade de significação

\section{B Utilidade dos prontuários para os usuários}

As primeiras Categorias obtidas a partir das Unidades de Significação acerca da percepção da utilidade dos prontuários para os USUÁRIOS foram:

- Informação oficial ou institucional;

- Informação pessoal;

- Segurança;

- Benefícios;
- Ética;

- Nenhuma utilidade.

As categorias INFORMAÇÃO OFICIAL OU INSTITUCIONAL E INFORMAÇÃO PESSOAL puderam ser associadas dando origem à categoria maior INFORMAÇÃO (o prontuário é útil para o usuário porque é fonte de informação para ele). As outras 4 categorias não responderam a nenhum critério que lhes permitissem associação.

Figura 2: Percepção da utilidade dos prontuários para os usuários de acordo com os Coordenadores dos CAPSi. Estado de São Paulo. 2009.

\begin{tabular}{lll}
\hline & INFORMAÇÃO & PESSOAL INSTITUCIONAL \\
\hline \multirow{2}{*}{ Usuário } & Segurança & \\
& Benefícios & \\
& Ética & \\
& Nenhuma & \\
\hline
\end{tabular}

Fonte: REIS AOA. Pesquisa Caracterização Epidemiológica e Sócio-demográfica dos Centros de Atenção Psicossociais Infantojuvenis do Estado de São Paulo, processo FAPESP 06/06902-2. 
Foram estabelecidas cinco categorias sem que se apreendesse um nexo lógico entre elas que permitisse suas associações mais particulares capazes de formar, desta sorte, categorias maiores. De modo similar, as freqüências de Unidades de Significação sob cada uma das subcategorias foram praticamente equivalentes. A única subcategoria que se diferenciou em significações diversificadas foi a denominada INFORMAÇÃO. Nesse caso, ela se bipartiu em "informação institucional" e "informação pessoal”.

Informação Institucional encerra unidades de significação que fazem referência à utilidade do prontuário para o usuário quando percebida como um veículo de comunicação para a Vara da Família, para o Conselho Tutelar ou para situações em que a criança ou o adolescente são objeto de transferência para uma outra instituição.

O segundo tipo, Informação Pessoal, refere-se às respostas conectadas com algum in- teresse de ordem não institucional e que interessa de maneira direta e imediata ao sujeito tal como "a mãe quer informação sobre a questão medicamentosa” ou ainda, "a mãe consegue os atestados que precisa”.

Optou-se por distinguir Informação das respostas que foram organizadas sob a rubrica "Benefícios" pelo fato desses últimos representarem uma situação muito específica em que a informação constitui um meio ou instrumento para obter alguma vantagem, tal como uma ajuda financeira ou algo concreto assemelhado. A categoria Segurança é da mesma ordem da categoria de mesmo nome e que se reporta à "utilidade para a equipe". Ética se refere ao fato de que as respostas, de caráter geral, respondem essencialmente, a um principio ético, sem mencionar nenhum benefício imediato e tangível, como por exemplo, "O Prontuário é do usuário". Finalmente, as respostas "não sei” ou "nenhuma" foram consideradas de mesma significação.

Figura 3: Percepção da utilidade dos prontuários para as equipes e os usuários de acordo com os Coordenadores dos CAPSI. Estado de São Paulo, 2009.

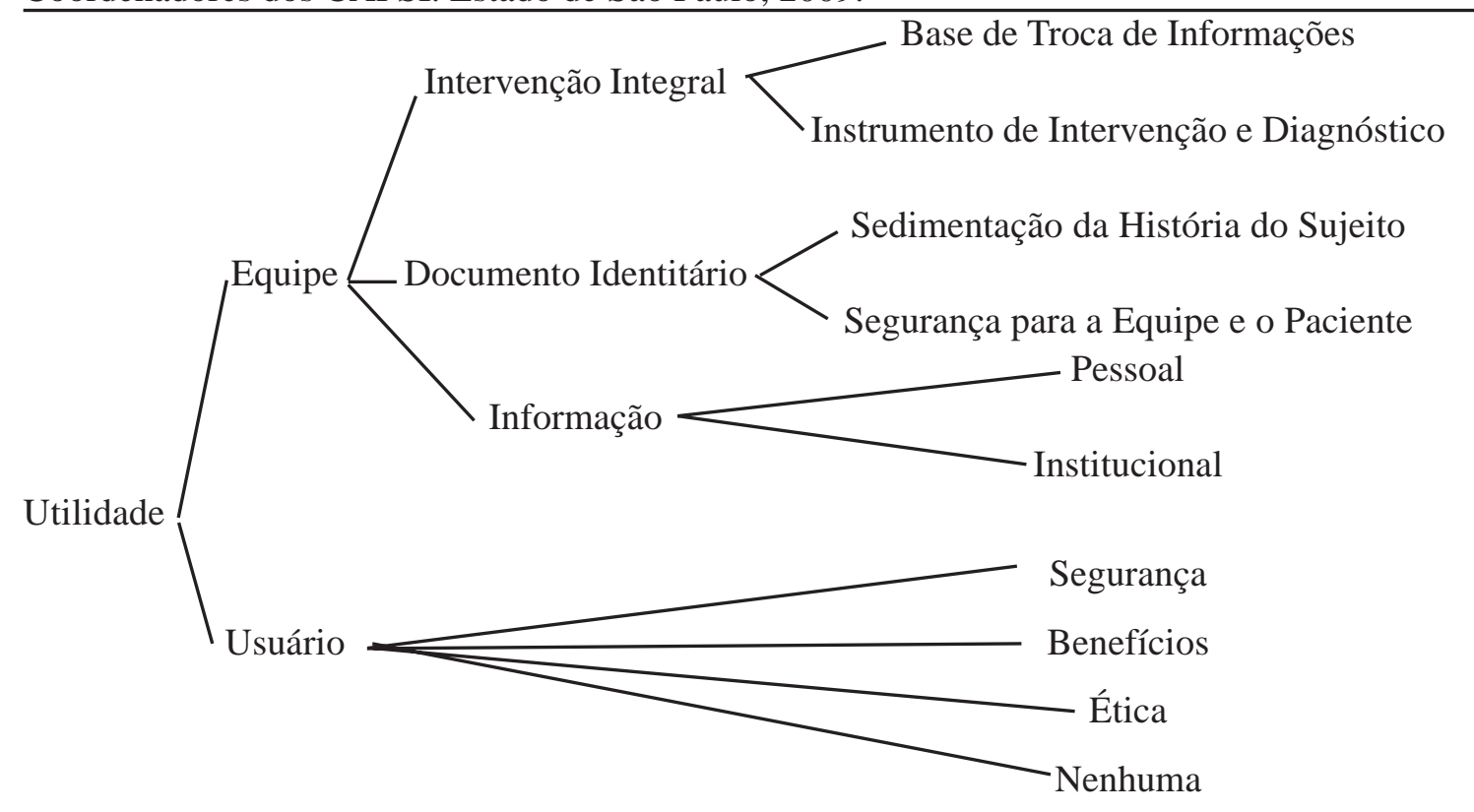

Fonte: REIS AOA. Pesquisa Caracterização Epidemiológica e Sócio-demográfica dos Centros de Atenção Psicossociais Infantojuvenis do Estado de São Paulo, processo FAPESP 06/06902-2 
Tabela 2: Distribuição do número e proporção das Unidades de Significação para as Categorias referentes à utilidade dos Prontuários na percepção dos Coordenadores dos CAPSi. São Paulo, 2009.

\begin{tabular}{lcc}
\hline CATEGORIA & $\mathbf{n}$ & $\mathbf{\%}$ \\
\hline Informação & 2 & 10,5 \\
Pessoal Institucional & 2 & 10,5 \\
Segurança & 3 & 15,8 \\
Benefício & 3 & 15,8 \\
Ética & 2 & 10,5 \\
Nenhuma & 7 & 36,9 \\
\hline Total & $\mathbf{1 9}$ & $\mathbf{1 0 0}$ \\
\hline
\end{tabular}

Fonte: REIS AOA. Pesquisa Caracterização Epidemiológica e Sócio-demográfica dos Centros de Atenção Psicossociais Infantojuvenis do Estado de São Paulo, processo FAPESP 06/06902-2.

As respostas em suas unidades de significação se distribuíram com freqüências aproximadamente assemelhadas pelas diversas categorias. A categoria NENHUMA, significando que o prontuário não representa utilidade alguma para o usuário é a única que se destaca com 36,9\% (n=7). Não deixa de ser surpreendente o fato de que o prontuário, percebido como tendo uma importância "fundamental", considerado como o "lugar da história do paciente" ou ainda visto como "chave mestra" se tratando de sua utilidade para a equipe técnica, seja quando se refere ao usuário, estimado como não tendo utilidade alguma. A assimetria entre esses dois pólos merece destaque e, possivelmente, aponta para a natureza da clínica que neles se processa. Poder-se-ia ainda dizer que a despeito dos imensos avanços realizados no plano ético e político no tangente aos prontuários, persiste uma certa inércia ideológica que perpetua na prática o entendimento de que esses instrumentos são voltados, no plano clínico, exclusivamente para a equipe técnica ou para o setor administrativo.

\section{CONSIDERAÇÕES FINAIS}

Os prontuários são percebidos pelos Coordenadores dos Centros de Atenção
Psicossocial do Estado de São Paulo como essenciais para o processo de intervenção clínica e acompanhamento das crianças e adolescentes. São, igualmente, vistos como dispositivo que possibilita a articulação dos membros das equipes técnicas dos CAPSi. Deste modo, eles são apreendidos como instrumentos cujos sentidos de intervenção apontam tanto para o "interior" do CAPSi quanto para o "exterior". No primeiro sentido, eles aparecem como ferramentas de suporte clínico que asseguram a continuidade da atenção dispensada às crianças e adolescentes em seus contextos. No segundo sentido, o prontuário é encarado como uma ferramenta de articulação da equipe. Por outro lado, não são percebidas quais ou qual a utilidade que o prontuário teria para o usuário, muito embora se compreenda que ele seja o lugar de inscrição da história dos sujeitos que são atendidos no CAPSi. A contradição de níveis de importância alocada à utilidade dos prontuários, ou seja, o desbalanço de sua utilidade quando se considera ora a equipe técnica ora os usuários do CAPSi enseja uma profunda discussão sobre os vieses da clínica praticada nos equipamentos de saúde mental. Provavelmente, uma visão legalista ou, no pior dos casos, decorrente de uma inércia conceitual que ainda percebe, a despeito da 
nova realidade da saúde mental, o prontuário como um instrumento da equipe técnica dos serviços de saúde impede que ele seja utilizado como um eficaz meio de diálogo, apetrecho clínico interativo entre a equipe e o usuário ${ }^{15}$. A reflexão, tendo por referência a utilidade e o valor dos prontuários, poderia não só realçar a importância de seu bom preenchimento como constituir-se como elemento de aprimoramento dos instrumentos que os CAPSi têm à disposição para perseguir sua finalidade de inclusão social que

\section{REFERÊNCIAS}

1. Goffman E. Manicômios, prisões e conventos. São Paulo: Ed. Perspectiva; 1974.

2. Goffman E. A representação do Eu na vida cotidiana. Petrópolis: Ed. Vozes; 1975.

3. Goffman E. Estigma.Rio de Janeiro: Ed. Zahar; 1975.

4. Laing D. Psiquiatria e Antipsiquitria. São Paulo: Perspectiva, 1967.

5. Cooper RD. The divided Self. London: Harmondsworth;1960.

6. Mannoni M. Le Psychiatre, son "fou" et la psychanalyse. Paris: Ed. Du Seuil; 1970.

7. Código de Ética Médico. Acesso em 02/ 03/2001. Online. Disponível na Internet:http://www.cfm.org.br/ codetic.htm.

8. Costa CGA. Desenvolvimento e avaliação tecnológica de um sistema de prontuário eletrônico do paciente, baseado nos paradigmas da World Wide Web e da Engenharia de Software [dissertação de mestrado]. Campinas: Universidade Estadual de Campinas; 2001.

9. Vasconcellos MM., Gribel EB, Moraes IHS. Registros em saúde: avaliação da passa pelo fato de fazer dos pacientes elementos ativos de seu contexto.

\section{AGRADECIMENTOS}

Agradecemos o apoio financeiro da Fundação de Amparo à Pesquisa do Estado de São Paulo (FAPESP) no projeto 2006/06902-2, intitulado Caracterização Epidemiológica e Sócio-Demográfica dos Centros de Atenção Psicossocial Infantojuvenis do Estado de São Paulo, relacionado a esse artigo.

qualidade do prontuário do paciente na atenção básica. Rio de Janeiro. Cadernos de Saúde Pública. 2008; 24 (1 Supl):173-182.

10. Novaes HMD. Pesquisa em, sobre e para os serviços de saúde: panorama internacional e questões para a pesquisa em saúde no Brasil. Cad. Saúde Publica Rio de Janeiro. 2004; 20 (2 Supl): 147157.

11. Conselho Regional de Medicina do Distrito Federal. Prontuário médico do paciente: guia para uso prático/Conselho Regional de Medicina do Distrito Federal. - Brasília: Conselho Regional de Medicina, 2006.

12. Bardin L. Análise de Conteúdo. Lisboa: Ed.70; 1979.

13. Creswell JW. Research design: Qualitative and quantitative approaches. Thousand Oaks, USA; London,UK; New Delhi,India: Sage Publications; 1994.

14. Franco MLPB. Análise do Conteúdo. Brasília: Plano editorial; 2004.

15. Moreno DMFC, Reis AOA. O momento da comunicação do resultado sorológico para o HIV sob a ótica winnicottiana. Pulsional Rev de Psicanálise. 2002; 156: 20-5.

Recebido em 22 de fevereiro de 2009. Modificado em 10 de julho de 2009. Aceito em 12 de agosto de 2009. 